\title{
Acute Myocardial Infarction in a 26-Year-0ld Man with Normal ST-Segment
}

\author{
Yuping Yang ${ }^{1}$, Hua Chen ${ }^{2 *}$ \\ ${ }^{1}$ Department of Cardiology, The Second Affiliated Hospital of Guilin Medical University, Guilin, China \\ ${ }^{2}$ Department of Emergency, The Second Affiliated Hospital of Guilin Medical University, Guilin, China \\ Email: yyp444@163.com, ^qylqjz@163.com
}

How to cite this paper: Yang, Y.P. and Chen, H. (2017) Acute Myocardial Infarction in a 26-Year-Old Man with Normal ST-Segment. Case Reports in Clinical Medicine, 6, 148-156.

https://doi.org/10.4236/crcm.2017.66015

Received: May 4, 2017

Accepted: June 20, 2017

Published: June 23, 2017

Copyright ( 92017 by authors and Scientific Research Publishing Inc. This work is licensed under the Creative Commons Attribution International License (CC BY 4.0).

http://creativecommons.org/licenses/by/4.0/

\section{cc) (i) Open Access}

\begin{abstract}
Acute myocardial infarction (AMI) is rare in young adults. The management of these patients is considered as a clinical challenge. We report the case of a 26-year-old man who was presented to the Emergency Room of Affiliated Hospital of Guilin Medical University with an acute onset of chest pain. Initially electrocardiogram (ECG) with no evidence of ST-segment abnormalities but QT prolongation a signal of sudden cardiac death, 20 minutes later, it revealed ST-segment elevation myocardial infarction (STEMI). Coronary angiography (CAG) demonstrated left main coronary artery occlusion. AMI was diagnosed based on clinical symptom, elevated cardiac biomarkers, electrocardiographic dynamic monitoring and CAG. The awareness of chest pain as possible underlying AMI symptom-especially in young patients presenting with QT prolongation-is crucial for clinical treatment, as a missed diagnosis can worsen the patient's further prognosis. In addition, reperfusion arrhythmia is a challenge to short-term outcomes of young patients with AMI, so it is necessary to make preoperative risk stratification.
\end{abstract}

\section{Keywords}

Acute Myocardial Infarction, Young Patient, Clinical Management, Reperfusion Arrhythmia

\section{Introduction}

Myocardial infarction (MI) is formed when there is complete interruption of blood flow to an area of myocardium. It involves necrosis of cardiac muscle followed by inflammatory cell infiltration and, because cardiac myocytes cannot regenerate eventual fibrous repair. The definition of MI published by the global Myocardial Infarction Task Force requires cardiac myocyte necrosis with a change in content of cardiac troponin ( $\mathrm{cTn}$ ) [1]. Patients with symptoms of 
myocardial ischemia, significant ST-segment/T-wave changes or left bundle branch block, the development of pathological Q waves, regional myocardium wall motion abnormality, should be highly suspected of having MI. On this basis, the plasma of cTn more than the 99th percentile normal reference limit is the key to diagnosing MI. Intracoronary thrombus identificated by coronary angiography (CAG) is the gold standard for the diagnosis of coronary heart disease. Electrocardiogram (ECG) as a basis noninvasive examination for diagnosis MI, ST-T wave criteria including: (1) New ST elevation at the J point in two contiguous leads with the cut-points: $\geq 0.1 \mathrm{mV}$ in all leads other than leads $\mathrm{V}_{2}-\mathrm{V}_{3}$ where the following cut points apply: $\geq 0.2 \mathrm{mV}$ in men $\geq 40 \mathrm{yr} ; \geq 0.25 \mathrm{mV}$ in men $<40 \mathrm{yr}$, or $\geq 0.15 \mathrm{mV}$ in women; (2) ST depression and T wave changes: New horizontal or down-sloping ST depression $\geq 0.05 \mathrm{mV}$ in two contiguous leads and/or $\mathrm{T}$ inversion $\geq 0.1 \mathrm{mV}$ in two contiguous leads with prominent $\mathrm{R}$ wave or $\mathrm{R} / \mathrm{S}$ ratio $>1$.

QT interval represents the total time of ventricular agitation, and it should be influenced by several factors such as gender, heart rate, conduction defects and underlying rhythm. Bazett's formula ( $\mathrm{QTC}=\mathrm{QT} / \sqrt{\mathrm{RR}}$ in seconds) as a universally adopted method provides an adequate correction for heart rate ranging anywhere between 60 and 100 beats/min. Both prolongation and shortening in the QT interval have been associated with ventricular tachy-arrhythmias, which predispose affected individuals to sudden cardiac death [2]. Attention to QTc may be a great significance to determine the prognosis of patients with acute myocardial infarction (AMI). Reperfusion arrhythmia, usually including ventricular tachycardia (VT) and ventricular fibrillation (VF), the arrhythmogenic mechanisms are related to the abrupt electrophysiological and biochemical changes brought about by the coronary revascularization [3].

In this case report, we aimed to describe the clinical features, treatment practices, and outcomes of young adults hospitalized with AMI.

\section{Case Report}

A 26-yr-old workman admitted to the emergency department with 16 hours of severe, central crusing chest pain. The pain radiated to his left arm and was accompanying with profuse sweating. He had no history of arterial arrhythmia, hypertension, or diabetic mellitus and no family history of similar complaints or sudden death. He smoked 20 cigarettes a day for 10 years. He was engaged in a high-pressure job. On physical examination, axillary temperature was $36.4^{\circ} \mathrm{C}$, respiratory rate was 21 breaths per minute, pulse rate was 81 beats per minute, blood pressure $115 / 91 \mathrm{mmHg}$, and oxygen saturation was $98 \%$. His first and second heart sounds were normal, and no appreciable murmur or pericardial friction rub could be heard.

Electrocardiogram (ECG) revealed a normal sinus rhythm with no ischemic change, however, it is worth noting that the QT interval corrected using the Bazett formula (QTc interval) was $530 \mathrm{~ms}$ (Figure 1(a)). On this basis, the ECG prompted QT prolongation. Transthoracic echocardiography revealed a left 


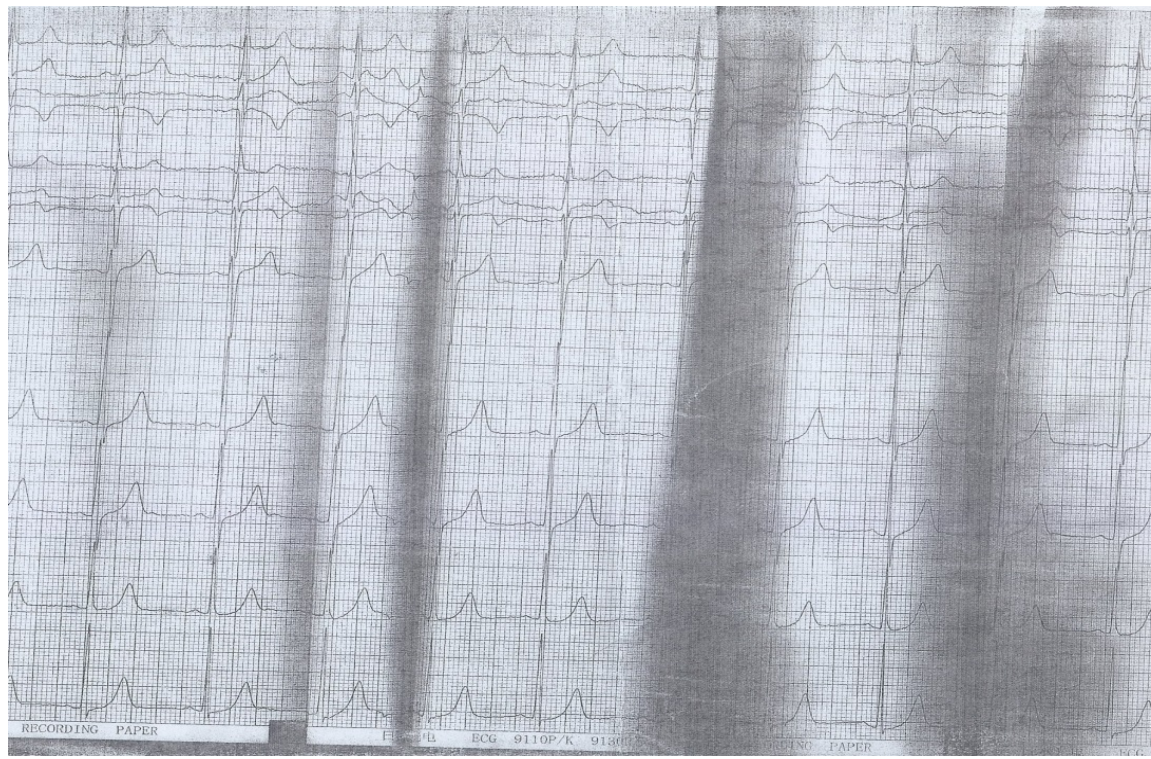

(a)

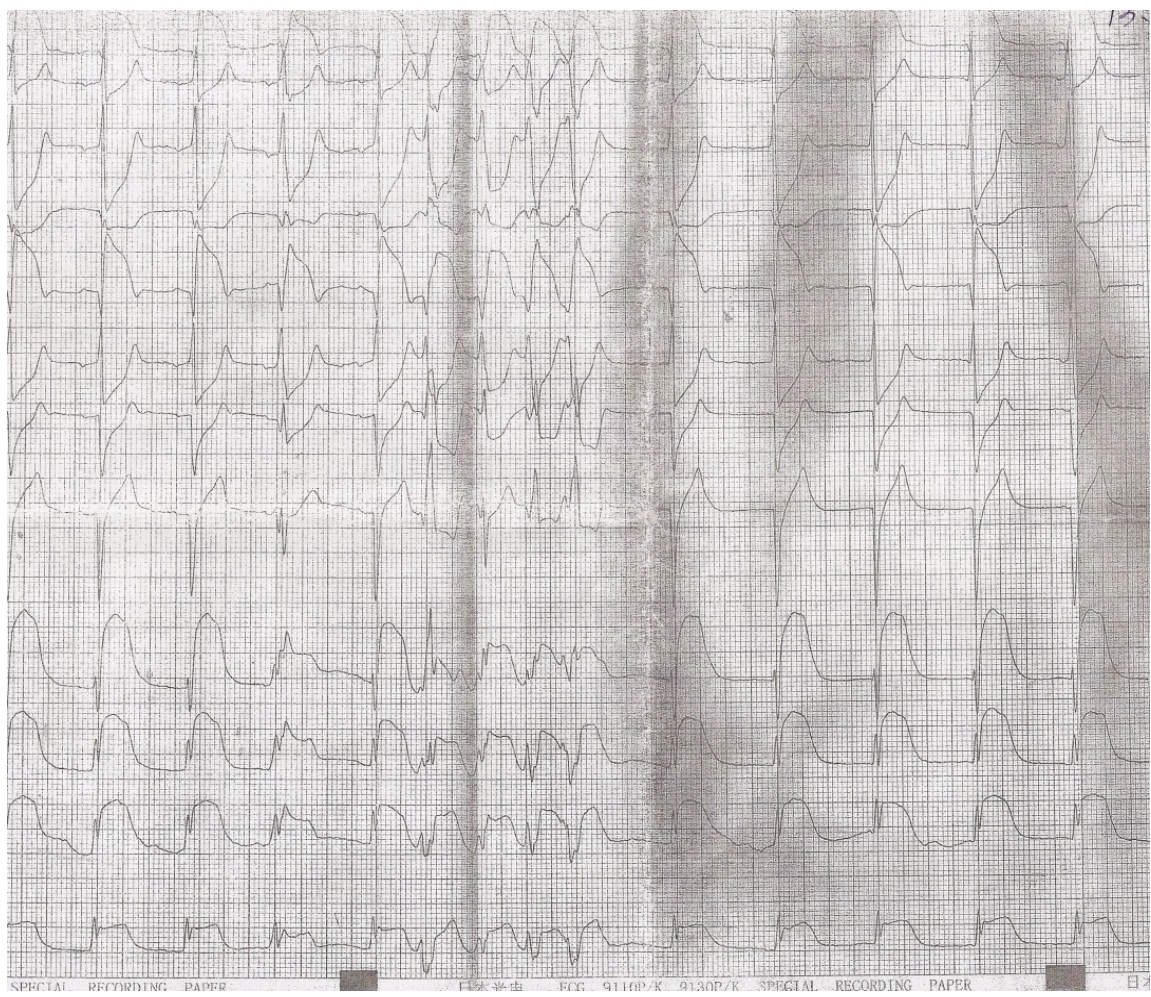

(b)

Figure 1. (a): Initial electrocardiogram showing normal sinus rhythm, ST segment normal, and QTc interval was $530 \mathrm{~ms}$. (b): Electrocardiogram showing ST segment elevations in leads $I, a V L$, and $V_{1}$ to $V_{6}$.

ventricular ejection fraction of 58\% with extensive wall motion abnormalities and mild tricuspid valve regurgitation (Figure 2). His emergent laboratory values (Table 1) demonstrated that high sensitivity troponin T (TnT-HS) far more than the 99th percentile normal reference limit. It illustrated that the young patient with an onset of chest pain suffered from an acute myocardial infarction. 


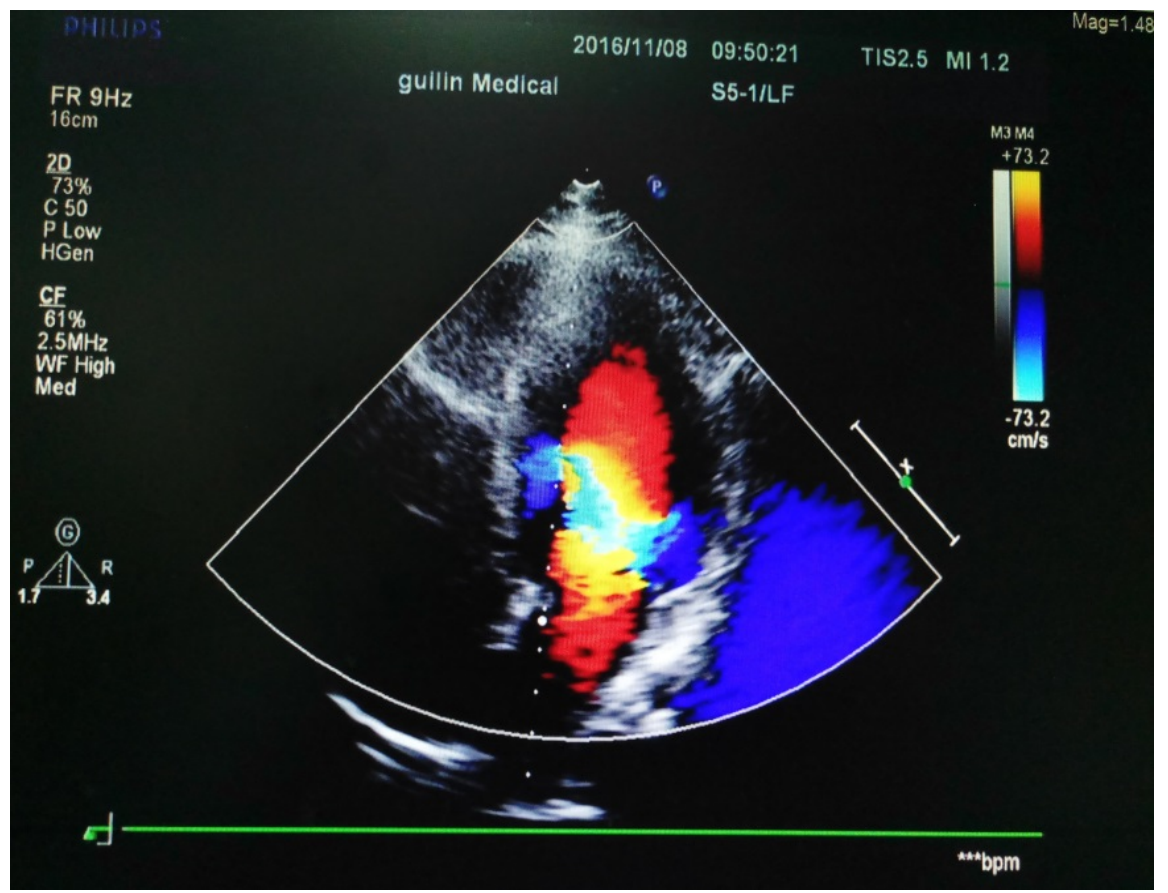

Figure 2. Transthoracic echocardiography revealed a left ventricular ejection fraction of $58 \%$ with extensive wall motion abnormalities and mild tricuspid valve regurgitation.

Table 1. Emergent laboratory values demonstrating hyperlipidemia and Myocardial injury markers increase.

\begin{tabular}{ccc}
\hline Laboratory test & Result & Normal values \\
\hline (Creatine Kinase) CK & 1148.00 & $25-192$ \\
(Creatine Kinase-MB) CK-MB & 154.27 & $0.001-24$ \\
(Triglyceride) TG & 2.76 & $0.23-1.71$ \\
(Cholesterin) CHOL & 6.45 & $3.6-6$ \\
(High-density lipoprotein c) HDLc & 0.95 & $0.7-2$ \\
(Low-density lipoprotein c) LDLc & 5.30 & $1.76-3.49$ \\
(Apolipoprotein AI) APO-AI & 1.24 & $1-1.6$ \\
(Apolipoprotein B) APO-B & 1.40 & $0.6-1$ \\
(High sensitivity troponin T) TnT-HS & 0.560 & $0.003-0.014$ \\
\hline
\end{tabular}

The patient was treated with aspirin $(300 \mathrm{mg})$, ticagrelor $(180 \mathrm{mg})$, and rosuvastatin calcium $(20 \mathrm{mg})$ in the emergency department which improved but did not completely alleviate his symptoms. Underwent electrocardiogram again at 20 minutes later, it revealed sinus rhythm with ST segment elevations in leads I, aVL, and $\mathrm{V}_{1}$ to $\mathrm{V}_{6}$ (Figure $1(\mathrm{~b})$ ). He was brought urgently to the cardiac catheterization laboratory. Suddenly, his expression is indifferent and presented with signs of cardiogenic shock. On examination, armpit temperature was 35.2, respiratory rate $20 / \mathrm{min}$, pulse rate $92 / \mathrm{min}$, and his blood pressure cannot be measured. Coronary angiography revealed an occlusion completely in the left main coronary artery with TIMI 0 flow (Figure 3(a)). During the process of coronary angiography, the patient performed Ventricular tachycardia. He was given intravenous amiodarone $(150 \mathrm{mg})$ to restore sinus rhythm. At the same 


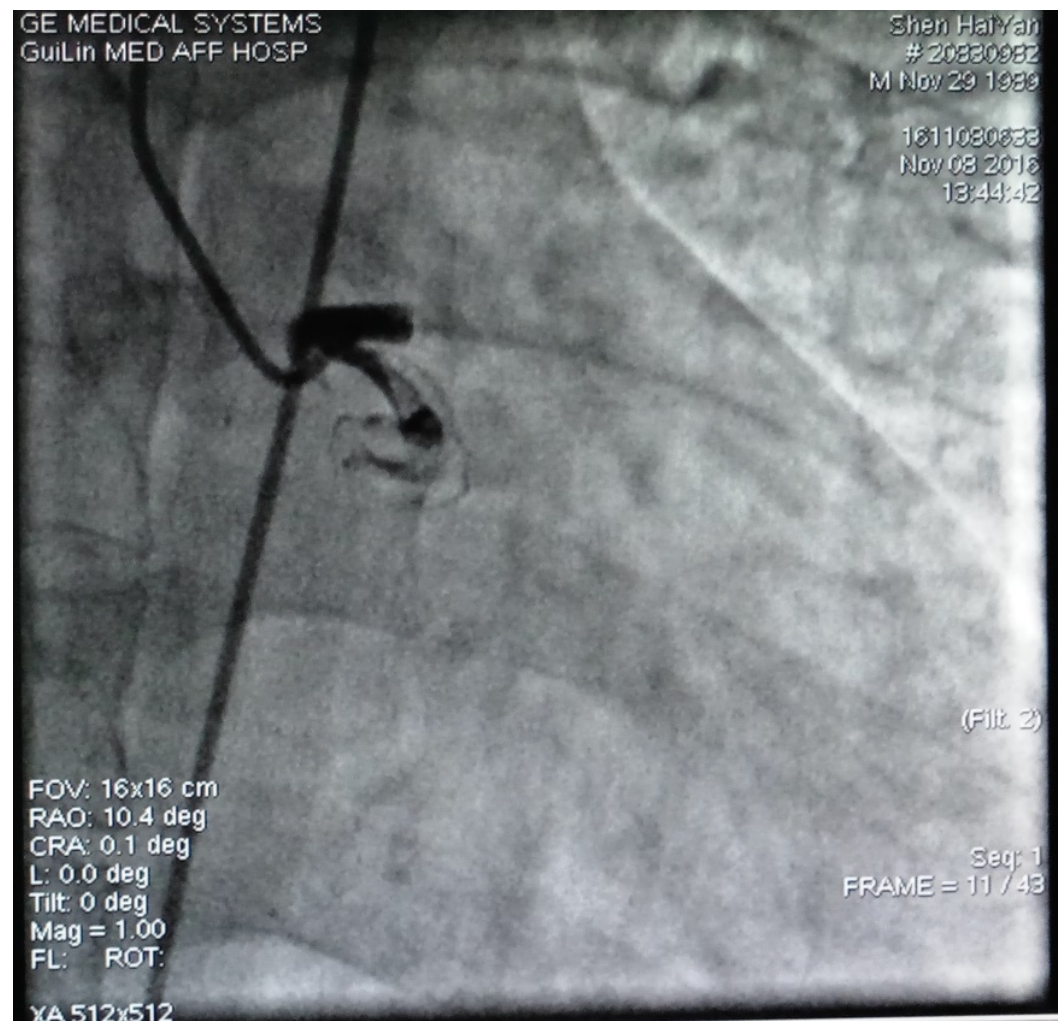

(a)

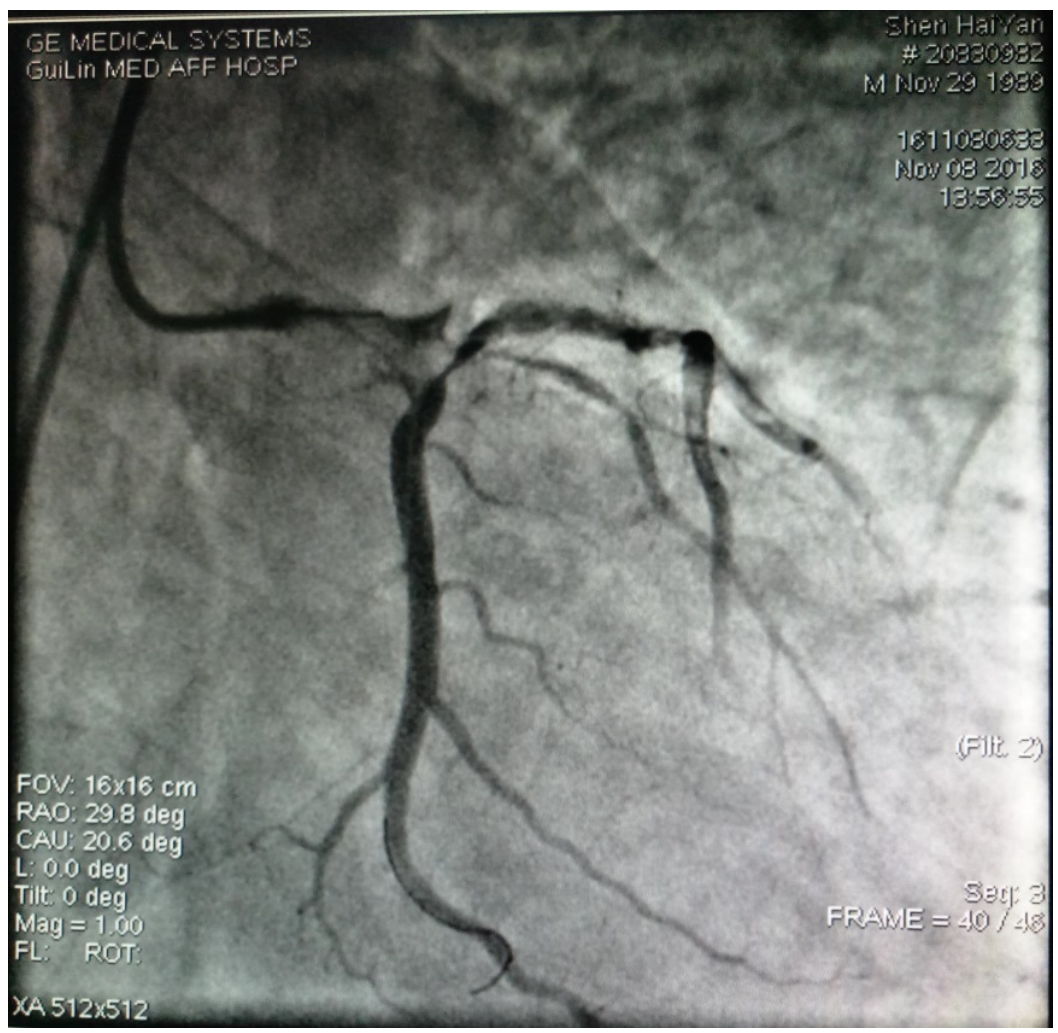

(b)

Figure 3. (a) Coronary angiography revealed an occlusion completely in the left main coronary artery with TIMI 0 flow. (b) After percutaneous balloon dilatation, the coronary angiography revealed left coronary artery flow reconstruction. 
time, he was successfully treated by percutaneous balloon dilatation of the left main coronary artery (Figure 3(b)). But 10 minutes later, he with an in-hospital ventricular fibrillation (VF) resulting in cardiac arrest was treated with cardiopulmonary resuscitation (CPR) and timely defibrillation, epinephrine, atropine, and sodium bicarbonate injection. Unfortunately, the patient died early after percutaneous coronary intervention.

\section{Discussion}

Acute myocardial infarction (AMI) with a increased incidence and disability is a significant economic burden to society public health. However, sparse data are available describing recent trends in the magnitude, incidence of morbidity, clinical features, treatment practices, and outcomes of comparatively young adults hospitalized with AMI. To the best of our knowledge, the lifestyles of young people, characterized by smoking, alcoholism, fast pace, high work stress, overeating, and overwork, likely cause disturbances in the internal environment disorder, such as fat metabolic disorder, platelet aggregation and coronary atherosclerosis, that increase the incidence of AMI. Those factors may cause coronary broken plaque or spasms in coronary arteries resulting in acute coronary ischemia and hypoxia. In addition, antecedent hyperlipidemia and antecedent hypertension are the other common risk factors [4]. Young patients with AMI tend to be predominantly male, smokers, and hyperlipidemic [5]. In our case report, the young patient's low-density lipoprotein c (LDLc) was above normal values (Table 1), which may bring about fat metabolic disorder.

For young patients with MI, especially in patients younger than 30 years old, clinicians should pay more attention to the history of previous diseases with high risk of thromboembolism, including nephrotic syndrome, antiphospholipid syndrome, disorders of glucose metabolism and abnormal coagulation, and they should actively promote prevention and the treatment of metabolic syndrome patients to reduce the incidence of complications of thromboembolism. Patients without traditional risk factors for the arterial thrombosis atherosclerosis, eight mutations and polymorphisms in six different genes were identified: factor II prothrombin, methylenetetrahydrofolate reductase, polymorphism of factor $\mathrm{V}$ Leiden, glycoprotein VI, and plasminogen activator inhibitor 1 polymorphism 4 G/5 G, which could be involved in the pathogenesis of atherosclerosis, and associated with unexpected coronary disease occurring at young age [6]. In our case report, the patient developing AMI at the age of 26 years with risk factors: smoking, work stress and hyperlipidemic. We are not sure whether the young patient's hyperlipidemic is congenital or not. Acute coronary syndrome (ACS) derived from the occlusion of coronary arteries, including unstable angina and AMI. ACS in young adults is a growing medical, public health, social, economical and psychological problem, related to unhealthy lifestyle and the prevalence of civilization-related diseases. The problem of ACS in young people as the available data mostly come from small series and case reports, often related to familial occurrence and genetic aspects familial occurrence of the disease [7]. 
ACS may be triggered by external activities; however, their frequency, predictors, and significance are uncertain. Predictors of a triggered ACS were age $<65$ years, elapsed angina, no former angiotensin-converting enzyme inhibitors/angiotens in 2 receptor blockers, impaired functional class, not having typical chest pain on presentation, and a final diagnosis of unstable angina [8].

For patients with an acute onset of chest pain, electrocardiogram (ECG) as a non-invasive test remains an essential tool in urgent management and risk stratification of patients with suspected AMI and should be acquired and interpreted within 10 min after patient admission [9]. ECGs should be acquired at 15 - 30 min intervals, especially if the initial ECG is equivocal and ECG must be interpreted with caution as it can, at times, be misleading, as in this case of AMI seen by us. In addition, QT interval have been associated with ventricular tachy-arrhythmias, which predispose affected individuals to sudden cardiac death, however, QT interval analysis is often overlooked. A corrected QT interval peak of at least $480 \mathrm{~ms}$ in the acute phase of ST-elevation myocardial infarction is an independent predictor of cardiovascular death [10]. The initial ECG of our young patient with no ischemic change, however, the QTc interval was $530 \mathrm{~ms}$ (Figure $1(\mathrm{a})$ ), which may harbinger cardiovascular death. In our case report, the young patient's emergent laboratory values demonstrated that he with an AMI. He was treated with aspirin, ticagrelor, and rosuvastatin calcium. At 20 minutes later, we underwent electrocardiogram again and it revealed ST segment elevations in leads $\mathrm{I}$, aVL, and $\mathrm{V}_{1}$ to $\mathrm{V}_{6}$. Compared with the prior ECG, wide spread and profound ST-T changes are associated with greater degrees of myocardial ischemia. However, we cannot determine whether the ECG changes were relevant with antiplatelet aggregation drug.

Sudden cardiac death (SCD) refers to an unexpected death from a cardiovascular cause that occurs rapidly outside of the hospital or in the emergency room. AMI has high mortality rate in patients with SCD, especially among men, and is the most common cause of death in Western countries [11]. Ventricular fibrillation (VF) is the primary rhythm resulting in SCD. The majority of VF events occurred during or after revascularization and may represent a different pathophysiologic process, as suggested by animal studies [12]. These independent risk factors of VF before primary percutaneous coronary intervention (PPCI) include age of $<60$ years, family history of SCD, use of statins, absence of preinfarction angina, alcohol intake $>7$ units per week, and history of AF. Patient with such risk factors may be particularly vulnerable to the proarrhythmic effects of ischemia and more likely to present with VF or SCD as their manifestation of CAD [13]. For people with AMI, rapid admission and acute interventional treatment combined with modern antithrombotic pharmacologic therapy frequently establish complete reperfusion and acutely stabilize the patient, but the reperfusion itself adds further to the damage in the myocardium compromising the longterm outcome [14]. As we found in our patient, after percutaneous balloon dilatation of the left main coronary artery, the coronary artery revascularization, the young patient ocurred VF. Although he was treated by timely defibrillation, our 
patient can be electrically converted to normal sinus and died. In patients with ongoing symptoms, significant hypotension, cardiogenic shock, and ECG evidence of large area of myocardium at risk and QT interval extended, more likely occur Ischemia-reperfusion arrhythmia after PCI, conservative treatment might be reasonable [15]. Missed the best time of ischemia and reperfusion for revascularization, may be faced with reperfusion arrhythmia, reperfusion injury and other adverse events, affecting the prognosis.

\section{Conclusion}

Acute myocardial infarction (AMI) is statistically uncommon in the young, and the current reported young myocardial infarction patients aged $40-50$ years. They usually have cardiovascular risk factors. Improved public health awareness and increasing use of efficacious therapies for AMI have likely contributed to the decline in mortality observed. This report underlines the need for rapid diagnosis and effective multimodal treatment setting for these patients. Dynamic monitoring of electrocardiogram (ECG) is important, especially if the initial ECG is equivocal. AMI was diagnosed based on clinical symptom, elevated cardiac biomarkers, ECG dynamic monitoring and coronary angiography. Reperfusion injury is believed to be a significant contributor to AMI and has become a major focus of research in recent years. In addition, how to reduce the incidence of ventricular fibrillation during or after revascularization is also a challenge to clinicians. Areas of active research and controversy include the development of best practices in screening, identification of young people at greatest risk and post-mortem evaluation.

\section{References}

[1] Thygesen, K., Alpert, J.S., Jaffe, A.S., Simoons, M.L., Chaitman, B.R. and White, H.D. (2012) Third Universal Definition of Myocardial Infarction. Nature Reviews Cardiology, 9, 620-633. https://doi.org/10.1038/nrcardio.2012.122

[2] Tse, G., Chan, Y.W., Keung, W. and Yan, B.P. (2017) Electrophysiological Mechanisms of Long and Short QT Syndromes. IJC Heart \& Vasculature, 14, 8-13.

[3] Siong, N.F., Shadi, I.T., Peters, N.S. and Lyon, A.R. (2013) Selective Heart Rate Reduction with Ivabradine Slows Ischaemia-Induced Electrophysiological Changes and Reduces Ischaemia-Reperfusion-Induced Ventricular Arrhythmias. Journal of Molecular and Cellular Cardiology, 59, 67-75.

[4] Wong, C.P., Loh, S.Y., Loh, K.K., Ong, P.J., Foo, D. and Ho, H.H. (2012) Acute Myocardial Infarction: Clinical Features and Outcomes in Young Adults in Singapore. World Journal of Cardiology, 4, 206-210. https://doi.org/10.4330/wjc.v4.i6.206

[5] Wang, Y.Y., Li, T., Liu, Y.W., Liu, B.J., Wang, Y., Hu, X.M., Li, X., Peng, W.J. and Li, J.F. (2014) Analysis of Risk Factors of ST-Segment Elevation Myocardial Infarction in Young Patients. BMC Cardiovascular Disorders, 14, 179.

https://doi.org/10.1186/1471-2261-14-179

[6] Dostálová, G., Bělohlávek, J., Hlubocká, Z., Bayerová, K., Bobčiková, P., Kvasnička, T., Kvasnička, J., Linhart, A. and Karetová, D. (2017) Multiple Thrombophilia Mutations as a Possible Cause of Premature Myocardial Infarction. Wien Klin Wochenschr. https://doi.org/10.1007/s00508-017-1193-Z 
[7] Maroszyńska-Dmoch, E.M. and Wożakowska-Kapłon, B. (2016) Clinical and Angiographic Characteristics of Coronary Artery Disease in Young Adults: A Single Centre Study. Kardiologia Polska, 74, 314-321.

https://doi.org/10.5603/KP.a2015.0178

[8] Tofler, G.H., Kopel, E., Klempfner, R., Eldar, M., Buckley, T., Goldenberg, I. and National Israel Survey of Acute Coronary Syndrome Investigators (2017) Triggers and Timing of Acute Coronary Syndromes. American Journal of Cardiology, 119, 1560-1565.

[9] Reddy, K., Khaliq, A. and Henning, R.J. (2015) Recent Advances in the Diagnosis and Treatment of Acute Myocardial Infarction. World Journal of Cardiology, 7, 243-276. https://doi.org/10.4330/wjc.v7.i5.243

[10] Galluzzo, A., Gallo, C., Battaglia, A.F., Canavosio, F.G., Botta, M., Bergerone, S. and Gaita, F. (2016) Prolonged QT Interval in ST-Elevation Myocardial Infarction: Predictors and Prognostic Value in Medium-Term Follow-Up. Journal of Cardiovascular Medicine, 17, 440-445. https://doi.org/10.2459/JCM.0000000000000317

[11] Risgaard, B., Nielsen, J.B., Jabbari, R., Haunsø, S., Holst, A.G., Winkel, B.G. and Tfelt-Hansen, J. (2013) Prior Myocardial Infarction in the Young: Predisposes to a High Relative Risk But Low Absolute Risk of a Sudden Cardiac Death. Europace, 15, 48-54. https://doi.org/10.1093/europace/eus190

[12] Andrag, E. and Curtis, M.J. (2013) Feasibility of Targeting Ischaemia-Related Ventricular Arrhythmias by Mimicry of Endogenous Protection by Endocannabinoids. British Journal of Pharmacology, 169, 1840-1848. https://doi.org/10.1111/bph.12252

[13] Jabbari, R., Engstrøm, T., Glinge, C., Risgaard, B., Jabbari, J., Winkel, B.G., Terkelsen, C.J., Tilsted, H.-H., Jensen, L.O., Hougaard, M., Chiuve, S.E., Pedersen, F., Svendsen, J.H., Haunsø, S., Albert, C.M. and Tfelt-Hansen, J. (2015) Incidence and Risk Factors of Ventricular Fibrillation Before Primary Angioplasty in Patients with First ST-Elevation Myocardial Infarction: A Nationwide Study in Denmark. Journal of the American Heart Association, 4, e001399. https://doi.org/10.1161/JAHA.114.001399

[14] Schmidt, M.R., Rasmussen, M.E. and Bøtker, H.E. (2017) Remote Ischemic Conditioning for Patients with STEMI. Journal of Cardiovascular Pharmacology and Therapeutics, 22, 302-309.

[15] Reddy, K., Khaliq, A. and Henning, R.J. (2015) Recent Advances in the Diagnosis and Treatment of Acute Myocardial Infarction. World Journal of Cardiology, 7, 243-276. https://doi.org/10.4330/wjc.v7.i5.243 
Submit or recommend next manuscript to SCIRP and we will provide best service for you:

Accepting pre-submission inquiries through Email, Facebook, LinkedIn, Twitter, etc. A wide selection of journals (inclusive of 9 subjects, more than 200 journals)

Providing 24-hour high-quality service

User-friendly online submission system

Fair and swift peer-review system

Efficient typesetting and proofreading procedure

Display of the result of downloads and visits, as well as the number of cited articles Maximum dissemination of your research work

Submit your manuscript at: http://papersubmission.scirp.org/

Or contact crcm@scirp.org 